\title{
Development of a novel classification system for anatomical variants of the puboprostatic ligaments with expert validation
}

\author{
Michael Kim, MSc, ; Shawna L. Boyle, MD; ${ }^{\dagger}$ Alfonso Fernandez, MD; ${ }^{\dagger}$ Edward D. Matsumoto, MD, MEd, FRCSC, \\ Dip ABU; Kenneth T. Pace, MD, MSc, FRCSC;: Maurice Anidjar, MD, PhD; Gregory N. Kozak, MD FRCSC,; \\ Sumit Davé, MD MCh; ;f Blayne K. Welk, MSc, MD, FRCSC; ${ }^{\dagger}$ Marjorie I. Johnson, PhD* \\ Stephen E. Pautler, BSc, MD, FRCSC ${ }^{\text {ta }}$
}

\begin{abstract}
*Division of Clinical Anatomy, Department of Anatomy \& Cell Biology, Western University, London, ON; 'Division of Urology, Department of Surgery, Western University, London, ON; §Division of Urology, Department of Surgery, McMaster University, Hamilton, ON; „Division of Urology, Department of Surgery, University of Toronto, Toronto, ON; *Division of Urology, Department of Surgery, McGill University, Montreal, QC; "Division of Urology, Department of Surgery, University of Calgary, Calgary, AB; ' EDivision of Urology, Department of Pediatrics, Western University, London, ON; aDivision of Surgical Oncology, Department of Oncology, Western University, London, ON
\end{abstract}

Cite as: Can Urol Assoc J 2014;8(11-12):432-6. http://dx.doi.org/10.5489/cuaj.1797 Published online December 15, 2014.

\section{Abstract}

Introduction: We propose a novel classification system with a validation study to help clinicians identify and typify commonly seen variants of the puboprostatic ligaments (PPL).

Methods: A preliminary dissection of 6 male cadavers and a prospective dataset of over 300 robotic-assisted laparoscopic radical prostatectomies (RARP) recorded on video were used to identify 4 distinct ligament types. Then the prospectively collected database of surgical videos was used to isolate images of the PPL from RARP. Over 300 surgical videos were reviewed and classified with 1 to 5 pictures saved for reference of the type of PPL. To validate the new classification system, we selected 5 independent, blinded expert robotic surgeons to classify 100 ligaments based on morphology into a 4-type system: parallel, V-shaped, inverted V-shape, and fused. One week later, a subset of 25 photographs was sent to the same experts and classified. Statistical analyses were performed to determine both the intra-rater and inter-rater reliability of the proposed system.

Results: Inverted V-shaped ligaments were noted most frequently (29.97\%), parallel and V-shaped ligaments were found at $19.19 \%$ and $11.11 \%$, respectively and fused ligaments were noted less frequently $(6.06 \%)$. There was good intra-rater agreement $(\kappa=0.66)$ and inter-rater agreement $(\kappa=0.67)$ for the classification system. Conclusions: This classification system provided standardized descriptions of ligament variations that could be adopted universally to help clinicians categorize the variants. The system, validated by several blinded expert surgeons, demonstrated that surgeons were able to learn and correctly classify the variants. The system may be useful in helping to predict peri- and postoperative outcomes; however, this will require further study.

\section{Introduction}

The puboprostatic ligaments $(\mathrm{PPL})$ anchor the prostate to the pubis and must be manipulated to successfully remove a cancerous prostate gland. There has been much debate regarding the course, composition, and origin of the PPL. Evidence indicates that the ligaments support the external urethral sphincter and help anchor the male membranous urethra in the pelvic floor. ${ }^{1}$ However, there appears to be no consensus as to whether or not the PPL are part of a muscle, or whether these ligaments run strictly to the prostate or extend to the bladder.

One school of thought proposes the PPL are condensations of endopelvic fascia that form a pyramid shape, with fibers fanning inferomedially from the pubic bone and attaching at the membranous urethra and the lateral aspect of the external striated urinary sphincter. ${ }^{2}$ In contrast, other studies have shown that the PPL should instead be termed the pubovesical ligaments, as the detrusor muscle runs from the urinary bladder down to the pubis as part of the ligaments. ${ }^{3}$ Histological evidence for this assertion was provided by Dorschner and colleagues; they showed that smooth muscle extended from the bladder down to the pubis. ${ }^{4}$

Although multiple studies have addressed the PPL and their manipulation during surgery, these studies have tended to provide only generalized descriptions and have neglected to mention the observed anatomical variations of the PPL. As there is currently no universally-accepted classification system for the PPL and their many variants, the aim of this study was to develop a simple but effective method of classification based on morphology of the ligaments. By designing a classification system for the variants, we expect that communication between urologists and other clinicians will be better facilitated. In addition to this benefit, we want to 
show that our classification system is valid and reproducible when used by urologic surgeons. The future application of this classification system looking at outcomes may help provide insight into some of the variation in outcomes, which does not seem to clearly relate to surgical or patient factors that are already known.

\section{Methods}

We first developed a classification system, then we validated this system.

\section{Development of classification system}

Six male cadaveric specimens of the pelvis were obtained through our university's Department of Anatomy \& Cell Biology. The cadavers had been previously embalmed and preserved, and the pelvic cavities already exposed prior to dissection. The pelvis was approached from a superior position and proceeded retropubically to best replicate the retropubic approach during robotic-assisted laparoscopic radical prostatectomies (RARP). However, the dissections were not done robotically mostly because the specimen provided was partially prosected, although the pelvis was intact. Pelvic fascia was cleared out until the PPL could be clearly visualized (Fig. 1), using the superficial dorsal vein as a central landmark.

Videos of RARPs recorded on digital video discs (DVDs) were obtained and viewed using Windows Media Player 12.0 (Microsoft Corporation, Redmond, WA). When the PPL were visualized onscreen, the video was paused and the "Print Screen" function was used to import the image of the PPL into Microsoft PowerPoint 2007 (Microsoft Corporation, Redmond, WA). The imported images were then cropped and enlarged before being saved as individual jpeg files. Resolution of the captured images ranged from $642 \times 481$ to $1118 \times 746$. The images of the PPL of each case were examined and classified based on morphology into a 4-class sys- tem: parallel, V-shaped, inverted V-shape, and fused (Fig. 2, Fig. 3). In instances where a classification was not possible (poor image quality; surgery was not properly recorded on video; video could not be found in the collection; ligaments were cut earlier in the surgery before recording started; ligaments could not be visualized clearly during viewing), a designation of not applicable (N/A) was given, and this represented $33.67 \%$ of the videos. The largest proportion of those was improper recording and inadequate view of the PPL during the dissection. These issues led to the adoption of a small pause and still frame collection during the operation in the ongoing prospective database collection.

In classifying the ligaments into different types in the original dataset, the morphology and course of the ligaments were examined. Parallel ligaments described left and right PPL that ran from the anterior surface of the prostate to the pubic symphysis in line and adjacent to one another (Fig. 2, part A and Fig. 3, part A). V-shaped ligaments originated at a more medial point on the prostate and diverged laterally before inserting onto the pubic bone and symphysis (Fig. 2, part B and Fig. 3, part B). Inverted V-shape ligaments originated at separate, distinct lateral points on the prostate before travelling medially to insert at a more medial point on the pubic bone (Fig. 2, part C and Fig. 3, part C). Finally, fused ligaments consisted of left and right ligaments that were indiscernible from each other; there were no clearly defined borders between the two ligaments, and for all intents and purposes, could be considered as one fused ligament (Fig. 2, part D and Fig. 3, part D). These descriptions were based on the appearance of the ligaments from an anterosuperior perspective (i.e., how a surgeon would view the ligaments in the retropubic space during a radical prostatectomy).

\section{Validation design}

A suitable sample size for validation design was calculated using a method outlined elsewhere. ${ }^{5}$ The calculation led to

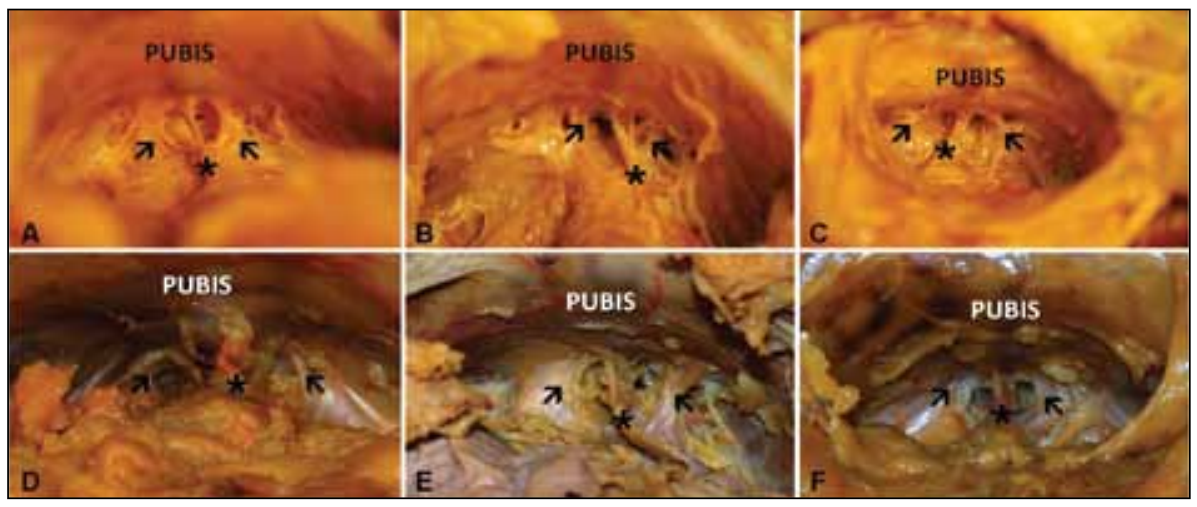

Fig. 1. Photographs of pelvic dissections of male cadaveric subjects $A-F$. The superficial dorsal vein is marked by the asterisk $(*)$, while the puboprostatic ligaments are highlighted by the arrows ( $\boldsymbol{\lambda})$. 
Kim et al.

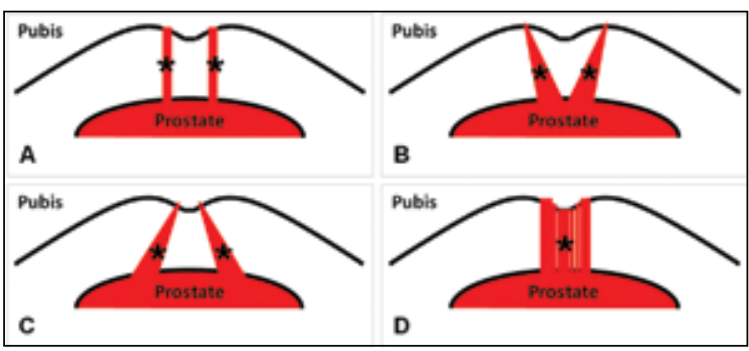

Fig. 2. A schematic of the four classification types of puboprostatic ligaments. A diagram outlining our proposed classification system for the puboprostatic ligament $(*)$ variations depicting $(A)$ parallel ligaments, (B) V-shaped ligaments, (C) inverted V-shaped ligaments, and (D) fused ligaments.

a sample size of, at minimum, 80 photographs with at least 4 surgeons classifying these photographs. To obtain an intrarater variability, we reclassified 20 of the original set. Five Canadian, academic urologic robotic surgeons familiar with the robotic view of the PPL were invited to complete the classification of 100 photographs and 1 week later, a subset of 25 from the original series. The 100 cases were selected, at random, from the original database of surgical videos which had previously agreed on classification by consensus of an endourology fellow, an anatomy Master's student, and a senior robotic urologic surgeon. The five experts were provided with a pictorial description of the classification system for reference and a scoring card of type 1, 2, 3, 4 or 0 for unclassifiable. All 5 experts completed both sets of classification and were blinded to the others and the database classification. They were unaware of the relative frequencies in the PPL types or the lack of any unclassified photographs in the sequence.

Statistical analyses were performed using the Statistical Analysis System (SAS) 9.2 (SAS Institute Inc., Cary, NC) and the Statistical Package for the Social Sciences (SPSS) 19.0 (IBM Corporation, Armonk, NY) software programs. Cohen's kappa coefficient calculations were performed for intra- and inter-rater agreement.

\section{Results}

Results of our dissections showed that variations in the PPL were visible even in embalmed, cadaveric specimens. We found evidence of parallel, V-shaped, and inverted V-shape

\begin{tabular}{lcc}
\hline \multicolumn{3}{l}{ Table 1. Experts' individual classification } \\
\hline Observer & Kappa & $\mathbf{9 5 \%} \mathbf{~ C l}$ \\
\hline Expert 1 & 0.772 & $0.668-0.877$ \\
Expert 2 & 0.772 & $0.655-0.889$ \\
Expert 3 & 0.707 & $0.592-0.821$ \\
Expert 4 & 0.485 & $0.334-0.635$ \\
Expert 5 & 0.614 & $0.478-0.749$ \\
Overall & 0.670 & \\
\hline
\end{tabular}

Cl: confidence interval.

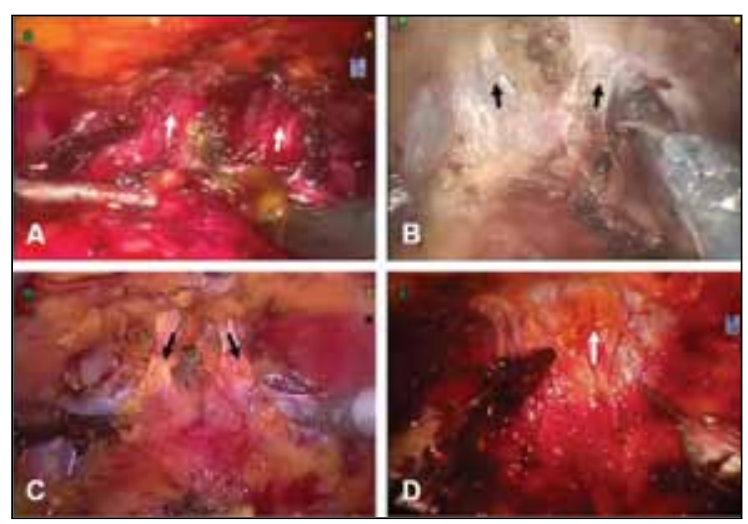

Fig. 3. Photographs of puboprostatic ligaments taken from video recordings of robot-assisted laparoscopic radical prostatectomies. (A) Parallel ligaments. (B) V-shaped ligaments. (C) Inverted $\mathrm{V}$-shaped ligaments. (D) Fused ligaments. Ligaments are highlighted by arrows ( $\boldsymbol{X})$.

ligaments in the 6 male cadavers (Fig. 1). However, fused ligaments were not present in any of the cadaveric specimens.

The original dataset of over 300 surgical videos was classified by an anatomy Master's student, a clinical endourology fellow and a senior urologic surgeon specializing in oncology and robotics. Based on the original dataset, we determined that inverted $\mathrm{V}$ ligaments were most frequent $(29.97 \%)$ and fused ligaments were least frequent $(6.06 \%)$, while parallel and V-shaped ligaments were found at $19.19 \%$ and $11.11 \%$, respectively (Fig. 3). One hundred cases $(33.67 \%)$ were deemed unclassifiable and excluded from our study. The dataset for the validation study was chosen, at random, from these surgical still shots representing the frequencies found in the total dataset.

The validation study tested the proposed classification system. This showed moderate inter-observer agreement based on the experts' classification $(\alpha=0.66)$ (Table 1$)$. There was also a moderate intra-rater agreement observed $(\kappa=0.66)$ (Table 2). ${ }^{6}$ After review of the database, it was noted that the experts agreed with each other but not the with the original dataset in $<10 \%$ of all items. These items were subsequently reclassified by expert consensus. All subsequent analyses were based on this final classification.

\begin{tabular}{lcc}
\hline \multicolumn{2}{c}{ Table 2. Experts' agreement on previous classification } \\
\hline Observer & Kappa & $\mathbf{9 5 \%} \mathbf{~ C l}$ \\
\hline Expert 1 & 0.767 & $0.563-0.971$ \\
Expert 2 & 0.658 & $0.411-0.904$ \\
Expert 3 & 0.647 & $0.406-0.888$ \\
Expert 4 & 0.402 & $0.143-0.662$ \\
Expert 5 & 0.804 & $0.605-1.000$ \\
Overall & 0.656 & \\
\hline
\end{tabular}

$\mathrm{Cl}$ : confidence interval. 


\section{Discussion}

Previous studies have mentioned PPL and the controversy surrounding both their naming and their role in return to continence and erectile function following radical prostatectomy. ${ }^{1,7}$ These studies, however, have not addressed the variations that exist in their morphologies. Much of the discussion has focused on whether or not the PPL are really pubovesical ligaments, ${ }^{7}$ whether saving them during surgery promotes an earlier return to continence, ${ }^{7-11}$ and whether or not sacrificing them will lead to reduced positive surgical margins. $9,10,12,13$ The focus of this study was not to address this controversy; instead, we proposed a system for classifying the variants of the ligaments that would facilitate communication and standardized descriptions among clinicians. The importance of standard nomenclature in surgical discussions is that using them as a landmark for dissection does not provide a consistent starting point and the underlying natural variation may change the outcomes. In fact, this natural variation may explain the conflicting evidence in PPL preservation related to continence, erectile dysfunction, and positive margin status.

The development of the PPL classification was based on observational data from surgical videos and anatomic cadaveric dissections. This system was developed by consensus and discussion of the anatomic variation and configuration. Our study has its limitations. It included surgeries were performed by a single surgeon at a single centre; also, the technical failure videos limited the actual sample size. However, with over 200 surgical videos and 6 cadaveric dissections, the anatomist and the clinical fellow had adequate observations to preliminarily design the classification. Further oversight by a senior urologic surgeon provided direction and experience.

Validation studies of our classification system showed that other experts were able to easily learn and apply the classification. This suggests that the observation of 4 distinct types is a true distinction with good inter-rater agreement $(\kappa=0.70)$. Although considered somewhat arbitrary, various guidelines have been published regarding the interpretation of Cohen's kappa coefficient. Landis and Koch suggested that values less than 0 indicated no agreement, $0.0-0.20$ poor agreement, $0.21-0.40$ fair agreement, $0.41-0.60$ moderate agreement, $0.61-0.80$ good agreement, and 0.81-1.0 very good agreement. ${ }^{6}$ Alternatively, Fleiss proposed guidelines recommending that kappa values below 0.40 indicate poor agreement, $0.40-0.75$ fair to good agreement, and over 0.75 as excellent agreement. ${ }^{14}$ According to both of these guidelines, our system shows good inter-rater agreement, suggesting that our proposed classification system is reliable. The intra-rater reliability also showed good agreement $(\alpha=0.67)$, suggesting that the system can be quickly adopted and easy to use. The original dataset provided 100 still shots, which were felt to fall into one of the 4 variants; however, some were "unclassified" by the experts. Based on this data, PPL can be difficult to categorize and this may be due to the retrospective screen shots as opposed to actual problems with classification. Some of the validating surgeons stated that classification of the PPL during surgery, rather than from the still shot, may be easier.

There were numerous challenging technical issues. During the surgeries, the camera was moved many times to provide the surgeon with the best possible view of the operating field. This changed the angle in which the raters were able to view the ligaments and could have made a ligament appear to be shaped differently than it really was. Agreeing on firm, anatomical landmarks to orient oneself could have alleviated this difficulty, while also reducing variability. In the future, it would also be beneficial to have the camera centre on the ligaments from the same angle each time, for a short period, to allow the raters to have a clear view of the ligaments and then to mark the ligaments for future viewing. Alternatively, rater could classify during surgery and assess the ligaments in real time. The degree of anatomic dissection can affect the appearance of the PPL and we attempted to capture the appearance prior to any dissection in the region of the ligaments to remove this as a potential confounder. Factors, such as gland size, degree of benign prostatic hyperplasia, glandular and pelvic configuration, could be potential confounders. Improvements to the study design and employing the aforementioned recommendations could ensure that future findings are applicable to the general population.

\section{Conclusion}

Our study showed that the proposed classification system of the PPL is viable and should be adopted to provide clinicians with a standardized method of categorizing variations. The proposed system is simple to use and that there is good inter- and intra-rater agreement. We believe that employing our classification system has the potential to improve our understanding of the anatomy and ultimately allow us to predict surgical outcomes and adverse events. This is worth pursuing in future research.

Competing interests: The authors declare no competing financial or personal interests.

This paper has been peer-reviewed.

\section{References}

1. Stolzenburg JU, Schwalenberg T, Horn LC, et al. Anatomical landmarks of radical prostatecomy. Eur Urol 2007:51:629-39. http://dx.doi.org/10.1016/i.eururo.2006.11.012 
Kim et al.

2. Steiner MS. The puboprostatic ligament and the male urethral suspensory mechanism: An anatomic study. Urology 1994;44:530-4. http://dx.doi.org/10.1016/50090-4295(94)80052-9

3. Myers RP. Detrusor apron, associated vascular plexus, and avascular plane: Relevance to radical retropubic prostatectomy - anatomic and surgical commentary. Urology 2002;59:472-9. http://dx.doi. org/10.1016/S0090-4295(02)01500-5

4. Dorschner $W$, Biesold $M$, Schmidt $F$, et al. The dispute about the external sphincter and the urogenital diaphragm. J Urol 1999;162:1942-5. http://dx.doi.org/10.1016/S0022-5347(05)68074-3

5. Sim J, Wright CC. The kappa statistic in reliability studies: use, interpretation, and sample size requirements. Phys Ther 2005:85:257-68.

6. Landis JR, Koch GG. The measurement of observer agreement for categorical data. Biometrics 1977;33:159-74. http://dx.doi.org/10.2307/2529310

7. Myers RP. Practical surgical anatomy for radical prostatectomy. Urol Clin North Am 2001;28:473-90. http://dx.doi.org/10.1016/S0094-0143(05)70156-7

8. Guillonneau B, el-Fettouh H, Baumert H, et al. Laparoscopic radical prostatectomy: Oncological evaluation after 1,000 cases at Montsouris Institute. J Urol 2003;169:1261-6. http://dx.doi.org/10.1097/01. ju.0000055141.36916.be

9. Lowe BA. Preservation of the anterior urethral ligamentous attachments in maintaining post-prostatectomy urinary continence: A comparative study. J Urol 1997;158:2137-41. http://dx.doi.org/10.1016/ S0022-5347(01)68180-1
10. Poore RE, McCullough DL, Jarow JP. Puboprostatic ligament sparing improves urinary continence after radical retropubic prostatectomy. Urology 1998;51:67-72. http://dx.doi.org/10.1016/S00904295(97)00479-2

11. Rassweiler J, Schulze M, Teber D, et al. Laparoscopic radical prostatectomy: Functional and oncological outcomes. Curr Opin Urol 2004; 14:75-82. http://dx.doi.org/10.1097/00042307-200403000-00005

12. Deliveliotis C, Protogerou V, Alargof E, et al. Radical prostatectomy: Bladder neck preservation and puboprostatic ligament sparing - effects on continence and positive margins. Urology 2002;60:855-8. http://dx.doi.org/10.1016/S0090-4295(02)01956-8

13. Katz R, Salomon L, Hoznek A, et al. Positive surgical margins in laparoscopic radical prostatectomy: The impact of apical dissection, bladder neck remodeling and nerve preservation. J Urol 2003; 169:2049-52. http://dx.doi.org/10.1097/01.ju.0000065822.15012.b7

14. Fleiss JL, Levin BA, Paik MC. Statistical methods for rates and proportions. New York: NY. John Wiley \& Sons; 2003. http://dx.doi.org/10.1002/0471445428

Correspondence: Dr. Stephen E. Pautler, Urology, St. Joseph's Hospital, 268 Grosvenor St., London, ON N6A 4V2; stephen.pautler@sihc.london.on.ca 